\title{
Análisis granulométrico para la producción indus trial del cobre a partir de la calcopirita
}

\section{RESUMEN}

En esta investigación se estudia el proceso de producción del cobre como producto principal y la calcopirita como producto alternativo. El proceso consiste en la disminución de tamaño a través de la molienda del mineral cuprífero hasta obtener un tamaño bastante fino, de manera que los granos de mineral de cobre queden sueltos.

El proyecto establece el análisis granulométrico a partir de la molienda del mineral sulfurado de cobre de calcopirita a nivel de laboratorio.

Se ha realizado Pruebas Experimentales de Molienda y Análisis Granulométrico de Mineral Sulfurado de Cobre, con la finalidad de determinar la molienda de un mineral sulfurado a escala de laboratorio y realizar las pruebas experimentales de molienda para un rango de tamaños de mineral en función del tiempo. Como se sabe, la liberación de los granos del mineral requiere normalmente una molienda hasta un tamaño, en el cual todas las partículas sean menores de 100 um de diámetro.

Palabras clave: Cinética de molienda, análisis granulométrico, calcopirita, cobre.

GRANULOMETRIC ANALYSIS FOR THE INDUSTRIAL PRODUCTION OF COPPER FROM CHALCOPYRITE

\section{ABSTRACT}

This research entitled "Particle size analysis for the industrial production of Chalcopyrite", was performed in the production process as the main product of copper and chalcopyrite as byproduct, the process treats about the reduction in size by milling of ore copper to a size fine enough so that the copper mineral grains are loose.

The Project establishes the Granulometric Analysis from the milling of sulfide mineral of Copper of Chalcopyrite to a Laboratory Scale.

It has been developed several experimental tests about milling and Granulometric Analysis of sulfide mineral of copper, with the aim of obtaining the milling of a sulfide mineral to a laboratory scale and carry out experimental tests of milling for a range of sizes of mineral in function of the time. In such manner, the reduction of the grains of mineral ask for a milling up to size, which every particles are minor of 100 um of diameter.

Keywords: Granulometric analysis, chalcopyrite, copper

\section{INTRODUCCIÓN}

El aislamiento de los minerales de cobre en forma de un concentrado requiere que el mineral sea triturado y molido finamente, de manera que los granos de dichos minerales se liberen de los granos de los otros minerales.

El grado necesario de molienda para que la liberación sea efectiva varía con los tamaños de los minerales; en esta investigación, se observa que dicho grado solamente puede ser determinado de manera confiable al llevar a cabo pruebas metalúrgicas de molienda [3].

Las dos razones para la existencia de un tamaño óptimo de partícula son:

- Las partículas excesivamente grandes provocan que los minerales de cobre se disimulen (entrelacen) en los minerales de la ganga, con lo que se evita su posterior tratamiento de concentración.[2]

- Las partículas excesivamente finas tienden a formar una lama, la cual cubre los minerales de cobre y disminuye la eficiencia con la cual se flota.[2]

La liberación de los granos de mineral requiere normalmente una molienda hasta un tamaño, en donde todas las partículas sean menores de 100 um de diámetro [3].

\section{FUNDAMENTO TEÓRICO}

La molienda es la operación final de reducción de tamaño o la liberación de las partículas valiosas del mineral, para proceder a su concentración. En esta etapa es necesario reducir su tamaño de 1", 3/4", 1/2", 3/8", 1/4", hasta un producto de 40 a $80 \%-200$ mallas.

\subsection{VARIABLES DE MOLIENDA}

Para que la molienda sea racional y económica hay que considerar las siguientes variables o factores:

Doctor en exploración y minería sostenible. Docente de la Facultad de Ingeniería Geológica, Minera, Metalúrgica, Geográfica. E-mail: aromerob@unmsm.edu.pe

2 Ingeniero metalúrgico. Docente de la Escuela de Minas. E-mail: sysyluz@hotmail.com

3 Ingeniero geográfico. Docente de la Facultad Ingeniería Geográfica. E-mail: warevalog@unmsm. edu.pe 


\section{Carga de mineral}

La cantidad de carga que se alimenta al molino debe ser controlada, procurando que la carga sea lo máximo posible. Si se alimenta poca carga se perderá capacidad de molienda y se gastará inútilmente bolas y chaquetas. Si se alimenta demasiada carga se sobrecargará el molino y al descargarlo se perderá tiempo y capacidad de molienda.

\section{Suministro de agua}

Cuando el mineral y el agua ingresan al molino forman un barro liviano llamado pulpa, que tiene la tendencia de pegarse a las bolas o barras, por otro lado el agua ayuda avanzar la carga molida.

Cuando la cantidad de agua es excesiva lava la barras o bolas, y cuando estás caen se golpean entre ellas y no muelen nada. Además el exceso de agua, saca demasiado rápido la carga y no da tiempo a moler, por lo que sale la carga gruesa.

Cuando hay poca agua la carga avanza lentamente y la pulpa se vuelve espesa alrededor de las barras o bolas, impidiendo buenos golpes porque la pulpa amortigua dichos golpes.

\section{Carga de bolas o barras}

Es necesario que el molino siempre tenga su carga normal de medios moledores, porque las barras y bolas se gastan y es necesario reponerlas. El consumo de las barras y bolas dependen del tonelaje tratado, dureza del mineral, tamaño del mineral alimentado y la finura que se desea obtener en la molienda. Diariamente, en la primera guardia debe reponerse el peso de bolas consumidas del día anterior.

Cuando el molino tiene exceso de bolas, se disminuye la capacidad del molino, ya que éstas ocupan el espacio que corresponde a la carga.

Cuando la carga de bolas está por debajo de lo normal, se pierde capacidad moledora porque habrá dificultad para llevar al mineral a la granulometría adecuada.

\section{Condiciones de los blindajes}

Es conveniente revisar periódicamente la condición en que se encuentran los blindajes, si están muy gastados ya no podrán elevar las bolas a la altura suficiente para que puedan trozar al mineral grueso.

La carga de bolas y la condición de los blindajes se puede controlar directamente por observación o indirectamente por la disminución de la capacidad de molienda y por análisis de mallas del producto de la molienda.

\section{Tiempo de molienda}

La permanencia del mineral dentro del molino determina el grado de finura de las partículas liberadas. El grado de finura está en relación directa con el tiempo de permanencia en el interior del molino. El tiempo de permanencia se regula por medio de la cantidad de agua añadida al molino.

La variable de operación principal para determinar la finura de la molienda es el número de veces que la mena pasa por los molinos. [4]

\subsection{CLASIFICACIÓN POR TAMAÑOS}

La molienda requiere cantidades considerables de energía eléctrica y tiempo; lo mejor es evitar moler más de lo necesario para obtener una recuperación satisfactoria de cobre. [1]

Las operaciones industriales de reducción de tamaño de partícula de costo mínimo, el cual es ligeramente más grande que el de la recuperación máxima. [3]

La reducción de tamaño se realiza teniendo en cuenta:

- Molienda en húmedo de las partículas trituradas en molino de bolas, mediante los cuales todas las fuerzas de abrasión, impacto y compresión contribuyen a quebrar la mena. [4]

- Una etapa importante en la molienda es tener certeza de que la descarga final del circuito de molienda sea de un tamaño de partícula suficientemente fino para lograr una concentración máxima. El material más grueso debe ser separado y regresado para que se vuelva a someter a molienda.

- Es en ese sentido que la clasificación se denomina a la separación de un conjunto de partículas de tamaños heterogéneos en dos porciones; es decir finos y gruesos. La clasificación por tamaños se efectúa mediante el empleo de una serie de tamices, que permiten determinar en qué malla se logro la mayor liberación de mineral de calcopirita. [3]

\section{DISEÑO EXPERIMENTAL}

Las pruebas experimentales se efectuaron en las instalaciones del Laboratorio de Metalurgia Extractiva y Planta JICA de la EAP Ing. Metalúrgica en los años 2006, 2007 y 2008. 
Por otra parte, el mineral de calcopirita pertenece a la zona del Complejo Marañón, el cual se sometió a un procesamiento del mineral: chancado, molienda y análisis granulométrico durante tiempos de molienda de 4, 8 y 12 minutos, con la finalidad de estudiar la cinética de molienda y ver el mayor grado de liberación que se dé en la malla.

Para el desarrollo del procedimiento experimental de la molienda y análisis granulométrico del mineral sulfurado de cobre, se tuvo en cuenta los siguientes materiales:

\section{EQUIPOS:}

- Chancadora de quijada. Es el equipo que permite la primera etapa de reducción de tamaño de la partícula del mineral en el rango de 3/8".

- Molino de bolas. Es el equipo que permite la segunda etapa de reducción de tamaño de partícula de mineral en el rango de tamaño de malla -10 .

- Mufla. Es el equipo que permite el secado de la pulpa de mineral obtenida, luego de la descarga del molino de bolas.

- Rot up. Es el nido de tamices, que permite la clasificación de tamaño de partículas de mineral molido según tamaño de abertura de los tamices empleados.

\section{MATERIALES:}

- Mineral: Sulfurado de cobre. Denominado también calcopirita.

- Mallas: 42,50,80,100,150,200,325,-325. Son los tamices que se emplearán para la clasificación del mineral, una vez que se haya realizado la molienda y el secado de la pulpa de descarga del molino.

- Espátula.

- Bandejas.

- Baldes

- Embudo

- Brocha.

\subsection{CARACTERIZACIÓN}

\subsubsection{Caracterización química del mineral de calcopirita}

El mineral de calcopirita pertenece a la zona del Complejo Marañón, el cual fue sometido a la caracterización química inicial. (Ver Tabla 1).
TABLA 1. ANÁLISIS QUIIMICO DE CALCOPIRITA

\begin{tabular}{|c|c|c|}
\hline $\mathrm{Cu}(\%)$ & $\mathrm{Fe}(\%)$ & $\mathrm{S}(\%)$ \\
\hline 10.5 & 18.55 & 26.35 \\
\hline
\end{tabular}

\subsection{ANÁLISIS GRANULOMÉTRICO DE MINERAL DE CALCOPIRITA}

a) Análisis granulométrico de mineral de calcopirita molido durante 4 minutos (Ver Tabla 2 y Gráfico 1).

Condiciones de la prueba:

Mineral: calcopirita.

Peso inicial de calcopirita: $994.9 \mathrm{gr}$

Peso final de calcopirita: $763.9 \mathrm{gr}$

\section{TABLA 2: ANÁLISIS GRANULOMÉTRICO DE MINERAL DE CALCOPIRITA PARA DETERMINAR LA LIBERACIÓN DE COBRE DURANTE 4 MINUTOS}

\begin{tabular}{|c|c|c|c|c|c|}
\hline \multirow{2}{*}{ Malla } & \multirow{2}{*}{$\begin{array}{c}\text { ABERTURA } \\
\text { (um) }\end{array}$} & \multicolumn{4}{|c|}{ MOLIENDA 4 MINUTOS } \\
\hline & & Peso & \%Peso & $\% A c(+)$ & $\% A c(-)$ \\
\hline 42 & 350 & 103.7 & 13.58 & 13.58 & 86.42 \\
\hline 50 & 297 & 31.9 & 4.18 & 17.75 & 82.25 \\
\hline 80 & 177 & 295.6 & 38.70 & 56.45 & 43.55 \\
\hline 100 & 149 & 10.3 & 1.35 & 57.80 & 42.20 \\
\hline 150 & 105 & 3.7 & 0.48 & 58.28 & 41.72 \\
\hline 200 & 74 & 176.5 & 23.11 & 81.38 & 18.62 \\
\hline 325 & 44 & 113.5 & 14.86 & 96.24 & 3.75 \\
\hline-325 & 44 & 28.7 & 3.76 & 100.00 & 0.00 \\
\hline \multicolumn{2}{|c|}{ Total } & 763.9 & 100.00 & & \\
\hline
\end{tabular}

Fuente: Pruebas de Laboratorio de Tesis de Flores S. "Tratamiento de Remediación de Efluentes Metalúrgicos con énfasis en el abatimiento de cobre con dolomita", para obtener Titulo Profesional de Ingeniera Metalúrgica de la UNMSM, 2009. [4]

Según el análisis granulométrico de mineral de calcopirita, el \%Ac(+), es decir, porcentaje de acumulado retenido en las mallas, el que presenta mayor valor es el de la malla 325 , cuyo valor es $96.24 \%$.

Por otra parte, el \%Ac(-), es decir el porcentaje de pasante acumulado en las mallas, que presenta mayor valor es el de la malla 42. No obstante, el rango de liberación de mineral durante la molienda está en el rango de malla -100

Es en ese sentido que el \%Ac(-) se dará en la malla $N^{\circ} 150$, cuyo valor será de $42.2 \%$. 


\section{GRÁFICO 1: CURVA DE ANÁLISIS GRANULOMÉTRICO DE CALCOPIRITA EN 4 MINUTOS}

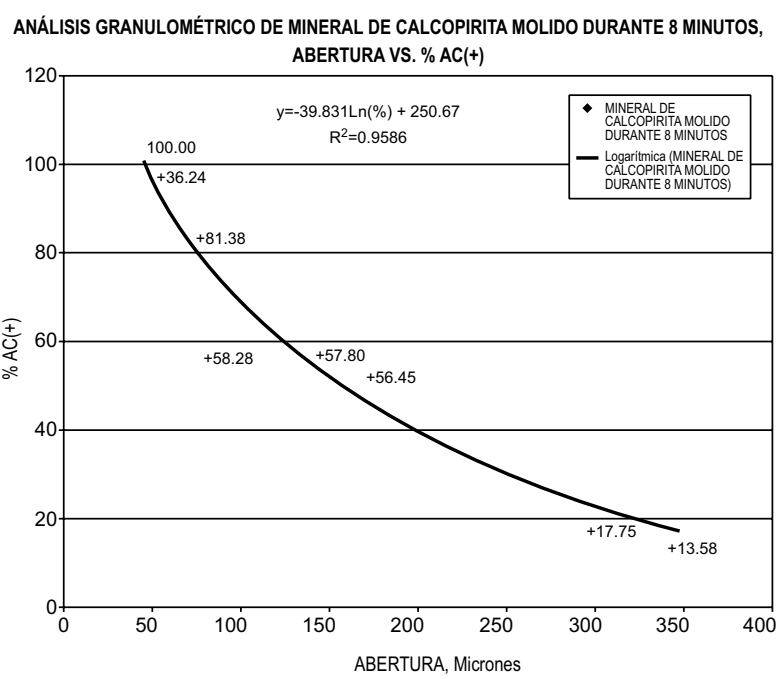

Fuente: Pruebas de Laboratorio de Tesis de Flores S. "Tratamiento de Remediación de Efluentes Metalúrgicos con énfasis en el abatimiento de cobre con dolomita", para obtener Título Profesional de Ingeniera Metalúrgica de la UNMSM, 2009. [4]

b) Análisis granulométrico de mineral de calcopirita molido durante 8 minutos. (Ver Tabla 3 y Gráfico 2).

Condiciones de la prueba:

Mineral: calcopirita

Peso inicial de calcopirita: $1000 \mathrm{gr}$

Peso final de calcopirita: $676 \mathrm{gr}$.

\section{TABLA 3: ANÁLISIS GRANULOMÉTRICO DE MINERAL DE CALCOPIRITA PARA DETERMINAR LA LIBERACIÓN DE COBRE DURANTE 8 MINUTOS}

\begin{tabular}{|c|c|c|c|c|c|}
\hline \multirow{2}{*}{ Malla } & \multirow{2}{*}{$\begin{array}{c}\text { ABERTURA } \\
\text { (um) }\end{array}$} & \multicolumn{4}{|c|}{ MOLIENDA 4 MINUTOS } \\
\hline & & Peso & \%Peso & $\% A c(+)$ & $\% A c(-)$ \\
\hline 42 & 350 & 14.4 & 2.13 & 2.13 & 97.87 \\
\hline 50 & 297 & 5.5 & 0.81 & 2.94 & 97.06 \\
\hline 80 & 177 & 338.3 & 50.04 & 52.99 & 47.01 \\
\hline 100 & 149 & 3.7 & 0.55 & 53.54 & 46.46 \\
\hline 150 & 105 & 11.8 & 1.75 & 55.28 & 44.72 \\
\hline 200 & 74 & 166.9 & 24.69 & 79.97 & 20.03 \\
\hline 325 & 44 & 118.9 & 17.59 & 97.56 & 2.44 \\
\hline-325 & 44 & 16.5 & 2.44 & 100.00 & 0.00 \\
\hline \multicolumn{2}{|c|}{ Total } & 676 & 100.00 & & \\
\hline
\end{tabular}

Fuente: Pruebas de Laboratorio de Tesis de Flores S. "Tratamiento de Remediación de Efluentes Metalúrgicos con énfasis en el abatimiento de cobre con dolomita", para obtener Título Profesional de Ingeniera Metalúrgica de la UNMSM, 2009. [4]
Según el análisis granulométrico de mineral de calcopirita, el \%Ac(+), es decir porcentaje de acumulado retenido en las mallas, el que presenta mayor valor es el de la malla 325 , cuyo valor es $97.56 \%$.

Por otra parte, el \%Ac(-), es decir el porcentaje de pasante acumulado en las mallas, que presenta mayor valor es el de la malla 50 . No obstante, el rango de liberación de mineral durante la molienda está en el rango de malla -100.

Es en ese sentido, que el \%Ac(-) se dará en la malla -100 , cuyo valor será de $46.46 \%$.

\section{GRÁFICO 2: CURVA DE ANÁLISIS GRANULOMÉTRICO DE CALCOPIRITA MOLIDA DURANTE 8 MINUTOS}

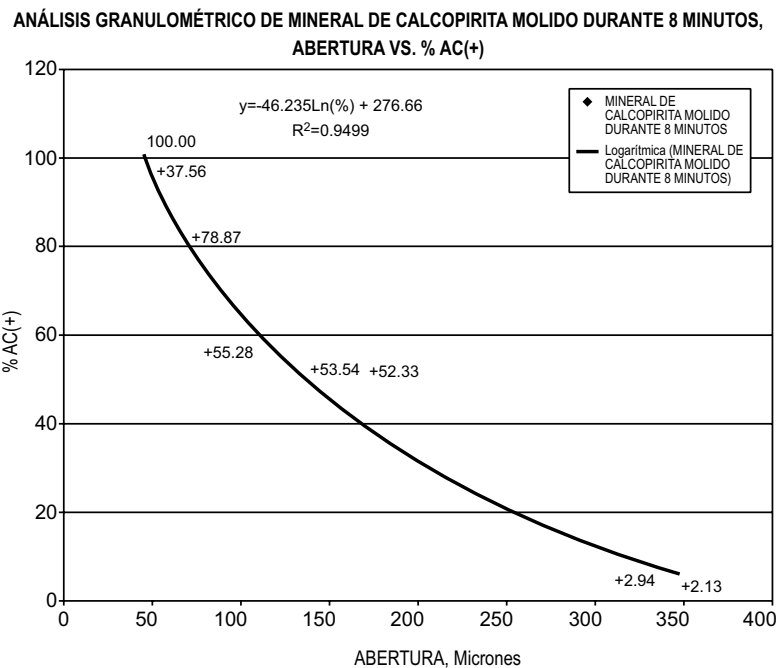

Fuente: Pruebas de Laboratorio de Tesis de Flores S. "Tratamiento de Remediación de Efluentes Metalúrgicos con énfasis en el abatimiento de cobre con dolomita", para obtener Título Profesional de Ingeniera Metalúrgica de la UNMSM, 2009. [4]

c) Análisis granulométrico de mineral de calcopirita molido durante 12 minutos. (Ver Tabla 4 y Gráfico 3).

Condiciones de la prueba:

Mineral: Calcopirita

Peso inicial de calcopirita: $1000 \mathrm{gr}$

Peso final de calcopirita: $589.2 \mathrm{gr}$ 


\section{TABLA 4: ANÁLISIS GRANULOMÉTRICO DE MINERAL DE CALCOPIRITA PARA DETERMINAR LA LIBERACIÓN DE COBRE DURANTE 12 MINUTOS}

\begin{tabular}{|c|c|c|c|c|c|}
\hline \multirow{2}{*}{ Malla } & \multirow{2}{*}{$\begin{array}{c}\text { ABERTURA } \\
\text { (um) }\end{array}$} & \multicolumn{4}{|c|}{ MOLIENDA 4 MINUTOS } \\
\hline & & Peso & \%Peso & $\% A c(+)$ & $\% A c(-)$ \\
\hline 42 & 350 & 3.7 & 0.63 & 0.63 & 99.37 \\
\hline 50 & 297 & 2.3 & 0.39 & 1.02 & 98.98 \\
\hline 80 & 177 & 241.5 & 40.99 & 42.01 & 57.99 \\
\hline 100 & 149 & 4.8 & 0.81 & 42.82 & 57.18 \\
\hline 150 & 105 & 6.6 & 1.12 & 43.94 & 56.06 \\
\hline 200 & 74 & 176.7 & 29.99 & 73.93 & 26.07 \\
\hline 325 & 44 & 132.6 & 22.51 & 95.44 & 3.56 \\
\hline-325 & 44 & 21 & 3.56 & 100.00 & 0.00 \\
\hline \multicolumn{2}{|c|}{ Total } & 589.2 & 100.00 & & \\
\hline
\end{tabular}

FuENTE: Pruebas de Laboratorio de Tesis de Flores S. "Tratamiento de Remediación de Efluentes Metalúrgicos con énfasis en el abatimiento de cobre con dolomita", para obtener Título Profesional de Ingeniera Metalúrgica de la UNMSM, 2009.[4]

Según el análisis granulométrico de mineral de calcopirita, el \%Ac(+), es decir porcentaje de acumulado retenido en las mallas, el que presenta mayor valor es el de la malla 325 , cuyo valor es $96.44 \%$.

Por otra parte, el \%Ac(-), es decir el porcentaje de pasante acumulado en las mallas, que presenta mayor valor es el de la malla 42. No obstante, el rango de liberación de mineral durante la molienda está en el rango de malla -100 .

Es en ese sentido que el \%Ac(-) se dará en la malla -100 , cuyo valor será de $57.18 \%$.
Alfonso A. Romero

\section{GRÁFICO 3: CURVA DE ANÁLISIS GRANULOMÉTRICO DE CALCOPIRITA MOLIDA DURANTE 12 MINUTOS}

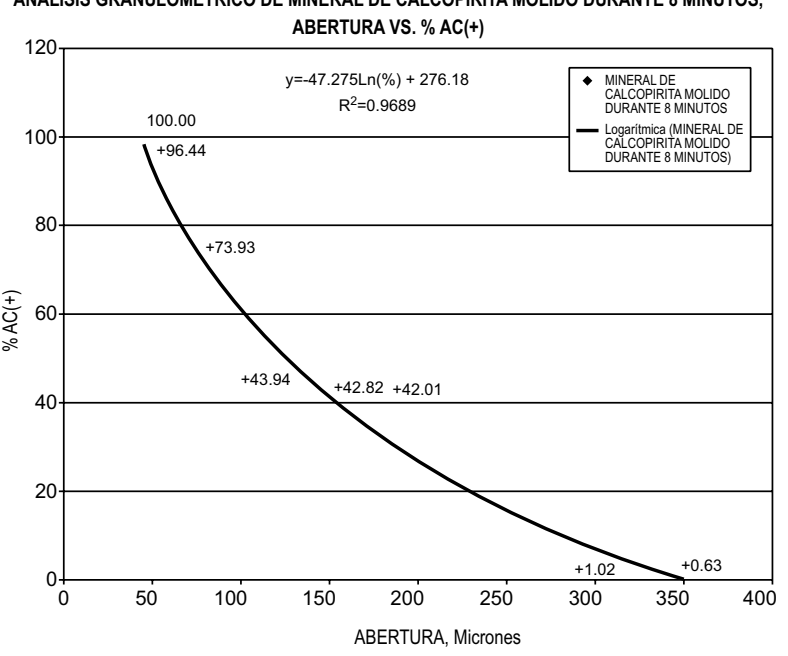

Fuente: Pruebas de Laboratorio de Tesis de Flores S. "Tratamiento de Remediación de Efluentes Metalúrgicos con énfasis en el abatimiento de cobre con dolomita", para obtener Titulo Profesional de Ingeniera Metalúrgica de la UNMSM, 2009.[4]

\section{DISCUSIÓN DE RESULTADOS}

Las pruebas experimentales de molienda y las curvas de análisis granulométrico de mineral sulfurado de cobre se realizan como un procedimiento estándar dentro la conminución de minerales, con la finalidad de determinar el grado de liberación de mineral de calcopirita.

En ese sentido, los resultados de las pruebas experimentales de análisis granulométrico señalan que existe un tamaño óptimo de partícula de mineral de calcopirita que se libera en mayor proporción en tiempos de molienda comprendidos entre 4 y 12 minutos, cuyo valor se da en la malla -100 .

Para el caso de análisis granulométrico de calcopirita molida durante 4 minutos, el valor de pasante acumulado en la malla -100 , es $42.20 \%$

Es importante afirmar que los minerales sulfurados de cobre, como la calcopirita, posee un rango de molienda comprendido entre 4 y 12 minutos.

Para el caso de análisis granulométrico de calcopirita molida durante 12 minutos, el valor de pasante acumulado en la malla -100 , es $57.18 \%$. 


\section{CONCLUSIONES}

Como se observa en el diseño experimental, la granulometría más adecuada para la recuperación óptima de cobre del mineral denominado calcopirita es de $56 \%$ Ac(-) tal como se ve en el Gráfico $N^{\circ} 3$, donde la malla es de -150 y el tiempo adecuado de molienda es de 12 minutos, cuyos datos deben tenerse en cuenta al momento de diseñar los equipos industriales de producción continua del cobre a partir de la calcopirita.

\section{REFERENCIAS BIBLIOGRÁFICAS}

[1] La Investigación e Innovación Tecnológica en la Minería del Cobre (2001). Comisión Chilena del Cobre Dirección de Estudios.
[2] Wilkomirsky, I. (1991). Metalurgia no ferrosa. Departamento de Ingeniería Metalúrgica, Universidad de Concepción.

[3] Biswas, A.K.; Davenport, W.K. (1994). El cobre metalurgia extractiva, Editorial Limusa.

[4] Flores Chávez, S. (2009). Tesis de Pregrado para obtener Titulo de Ingeniero Metalúrgico, Universidad Nacional Mayor de San Marcos; "Tratamiento de remediación de efluentes metalúrgicos con énfasis en el abatimiento de cobre con dolomita". 